Bulletin of the Section of Logic

Volume 51/1 (2022), pp. 57-71

https://doi.org/10.18778/0138-0680.2021.20

\title{
A NOTE ON THE INTUITIONISTIC LOGIC OF FALSE BELIEF
}

\begin{abstract}
In this paper we analyse logic of false belief in the intuitionistic setting. This logic, studied in its classical version by Steinsvold, Fan, Gilbert and Venturi, describes the following situation: a formula $\varphi$ is not satisfied in a given world, but we still believe in it (or we think that it should be accepted). Another interpretations are also possible: e.g. that we do not accept $\varphi$ but it is imposed on us by a kind of council or advisory board. From the mathematical point of view, the idea is expressed by an adequate form of modal operator $W$ which is interpreted in relational frames with neighborhoods. We discuss monotonicity of forcing, soundness, completeness and several other issues. Finally, we mention the fact that it is possible to investigate intuitionistic logics of unknown truths.

Keywords: Intuitionistic modal logic, non-normal modal logic, neighborhood semantics.

2020 Mathematical Subject Classification: 03B45, 03B20, 03A10.

\section{Preliminaries}

Logic of false belief was studied e.g. by Steinsvold [3], Gilbert and Venturi [2] or Fan [1]. Those authors obtained several interesting results concerning completeness and expressivity. Their propositional systems were based on classical modal logics (i.e. with the law of the excluded middle). As for the semantics, they used relational (Kripke) and neighborhood frames.
\end{abstract}

Presented by: Revantha Ramanayake

Received: October 15, 2020

Published online: September 1, 2021

(C) Copyright by Author(s), Łódź 2022

(C) Copyright for this edition by Uniwersytet Łódzki, Łódź 2022 
In general, the idea is to describe the following situation: $\varphi$ is false but it is still believed (see [2]). This concept is expressed by the very definition of forcing: if $w$ is a possible world then $w \Vdash \mathrm{W} \varphi \Leftrightarrow w \nVdash \varphi$ and $V(\varphi) \in \mathcal{N}_{w}$. The fact that $\varphi$ is erroneously taken for true, is modelled by the second part of this definition: we see that $V(\varphi)$ is among our neighborhoods.

Other interpretations are also possible. For example, we deny $\varphi$ (in a given world) but it is imposed on us by a kind of council or advisory board. We are encouraged to accept $\varphi$, at least in a particular world, because the set of all worlds accepting $\varphi$ is one of our neighborhoods. This means that $V(\varphi)$ gathers worlds (and thus situations or circumstances) which are similar to our present situation, hence maybe we should rethink our opinion on $\varphi$. Also, we can identify possible worlds with different people, accepting (or not) various formulas. Then $w$-neighborhoods can be considered as (more or less) credible groups of advisors or lustrators.

We can assume that our worlds are pre-ordered and if $w \leq v$, then $v$ accepts at least everything which was previously approved by $w$, i.e. lower located worlds have certain influence on the upper worlds. In some sense, we get a hierarchy of information and credibility. Now our model becomes intuitionistic: we have persistence of truth (with respect to $\leq$ ). This approach will be studied in the present paper. We are interested mostly in completeness and monotonicity of forcing. We show several intuitionistic versions of classical false belief systems. We discuss restrictions which can or have to be imposed on neighborhoods. We also point out some subtle limitations and advantages of intuitionistic framework in the context of minimal, maximal and intermediate canonical models. Finally, we make some comments on the intuitionistic logic of unknown truths. Classical systems of this kind are often examined together with logics of false belief. We show some general ideas, difficulties and suppositions.

\section{Logic of false belief}

\subsection{Alphabet and language}

Our logic is propositional, without quantifiers. We introduce the alphabet of our language below. 
DEFinition 2.1. $\mathbf{i E}^{\mathrm{W}}$-alphabet consists of:

1. $P V$ which is a fixed denumerable set of propositional variables $p, q, r, s, \ldots$

2. Common logical connectives and constants which are $\wedge, \vee, \perp$ and $\rightarrow$.

3. The only derived connective which is $\neg$ (thus $\neg \varphi$ is a shortcut for $\varphi \rightarrow \perp)$.

4. One modal operator: W.

Well-formed formulas are built recursively in a standard style: if $\varphi, \psi$ are $w f f$ 's then also $\varphi \vee \psi, \varphi \wedge \psi, \varphi \rightarrow \psi$ and $\mathrm{W} \varphi$. Note that $\Leftarrow, \Rightarrow$ and $\Leftrightarrow$ are used only on the level of meta-language (which is classical).

\subsection{Structures and models}

Our initial structure is a pre-ordered neighborhood frame (pn-frame) defined as follows:

Definition 2.2. pn-frame is a triple $F=\langle W, \mathcal{N}, \leq\rangle$ where $\leq$ is a partial order on $W$ and $\mathcal{N}$ is a function from $W$ into $P(P(W))$.

This definition is very general and it does not provide any relationship between $\leq$ and $\mathcal{N}$. In particular, it will not allow us to speak about monotonicity of forcing with respect to modal formulas. Thus, we shall introduce a particular subclass of $\mathbf{p n}$-frames.

DEFINITION 2.3.

$\mathbf{i E}^{\mathrm{W}} \mathbf{p n}$-frame is a $\mathbf{p n}$-frame with the following additional restriction:

$$
\left[w \leq v, X \in \mathcal{N}_{w}, v \notin X\right] \Rightarrow X \in \mathcal{N}_{v} .
$$

Having structures with appropriate features, we may introduce the notion of model. The first one is general and can be considered as a pattern for the further development of particular models.

Definition 2.4. A pn-model is a quadruple $M=\langle W, \mathcal{N}, \leq, V\rangle$ where $\langle W, \mathcal{N}, \leq\rangle$ is a pn-frame and $V$ is a function from $P V$ into $P(W)$ such that: if $w \in V(q)$ and $w \leq v$ then $v \in V(q)$. 
Definition 2.5. For every pn-model $M=\langle W, \mathcal{N}, \leq, V\rangle$, forcing of formulas in a world $w \in W$ is defined inductively:

1. $w \nVdash \perp$.

2. $w \Vdash q \Leftrightarrow w \in V(q)$ for any $q \in P V$.

3. $w \Vdash \varphi \wedge \psi($ resp. $\varphi \vee \psi) \Leftrightarrow w \Vdash \varphi$ and (resp. or) $w \Vdash \psi$.

4. $w \Vdash \varphi \rightarrow \psi \Leftrightarrow v \nVdash \varphi$ or $v \Vdash \psi$ for each $v \in W$ such that $w \leq v$.

We do not make difference between primal valuation and its extended version, using only one symbol $V$. Let us use shortcut $V(\varphi)$ for $\{z \in W$; $z \Vdash \varphi\}$.

Remark 2.6. Of course, the definition above allows us to say that $w \Vdash \neg \varphi$ $\Leftrightarrow$ for any $v \geq w, v \nVdash \varphi$.

Again, we narrow down our initial definition:

Definition 2.7. An $\mathbf{i E}{ }^{\mathrm{W}} \mathbf{p n}$-model is a $\mathbf{p n - m o d e l ~ w i t h ~ v a l u a t i o n ~ a n d ~ f o r c - ~}$ ing of non-modal formulas defined just like in Def. 2.5 but with an additional clause:

$w \Vdash \mathrm{W} \varphi \Leftrightarrow w \Vdash \neg \varphi$ and $V(\varphi) \in \mathcal{N}_{w}$.

\subsection{Monotonicity of forcing}

Here we prove the following fact:

TheOREM 2.8. In every $\mathbf{i E}^{\mathrm{W}} \mathbf{p n}$-model $M=\langle W, \mathcal{N}, \leq, V\rangle$ the following holds: if $w \Vdash \gamma$ and $w \leq v$, then $v \Vdash \gamma$.

Proof: We shall discuss only the modal case. Assume that $\gamma=\mathbf{W} \varphi$, $w, v \in W, w \leq v$ and $w \Vdash \gamma$. Hence, $w \Vdash \neg \varphi$ and $V(\varphi) \in \mathcal{N}_{w}$. Of course $v \Vdash \neg \varphi$. In particular, it means that $v \notin V(\varphi) \in \mathcal{N}_{w}$. Now Cond. 2.1 allows us to say that $V(\varphi) \in \mathcal{N}_{v}$. Hence, $w \Vdash \mathrm{W} \varphi$.

There is a difference between our definition of $W$ and the one presented by the authors in [1] or [2]. Their operator was defined as follows: $w \Vdash \mathrm{W} \varphi$ $\Leftrightarrow w \nVdash \varphi$ and $V(\varphi) \in \mathcal{N}_{w}$. However, their framework was classical, so there was no difference between lack of acceptance and acceptance of negation. In our intuitionistic setting, this approach would be problematic: if $w \nVdash \varphi$, then it does not mean that $\varphi$ is denied in each (or even in one) world $v$ 
placed above $w$. In fact, there is no reason for it: this is the whole difference between classical and intuitionistic negation, at least from the semantical point of view. Hence, we had to modify the interpretation of $\mathrm{W}$.

One could say that there is no need to use pre-orders. Maybe we should express everything in the language of neighborhoods? In fact, we have already established pure neighborhood semantics for intuitionistic modal $\operatorname{logics}^{1}$. However, it was applicable for normal modal logics. It was based on the assumption that minimal neighborhood (corresponding to the intuitionistic pre-order) is always contained in the maximal one (the idea was that forcing of $\square \varphi$ in a given world $w$ is equivalent with its forcing in any world from $\bigcup N_{w}$ ). In case of weak modal logics it would not be relevant. Of course, we may easily replace $\leq$ with neighborhoods but we think that it would lead us to the concept of two neighborhood families (one "intuitionistic" and one "modal"). This can be made but it would be rather a matter of notation and some aesthetic preferences.

Note that the concept of pre-ordered neighborhood model (for weak intuitionistic modal logics) was used (for example) by Dalmonte et al. in [4]. They used pre-order to speak about monotonicity of forcing - and neighborhoods to speak about modalities (well, they used two families of neighborhoods but one of them was connected with $\square$ and the other one with $\diamond$, none of them modelled pre-order which was, as we said, directly introduced).

\subsection{Axiomatization}

In this subsection we present sound and complete axiomatization of our basic system.

Definition 2.9. $\mathbf{i E}^{\mathrm{W}}$ is defined as the smallest set of formulas containing IPC $\cup\{\mathrm{WE}\}$ and closed under the following set of inference rules: \{MP , REW \}, where:

1. IPC is the set of all intuitionistic axiom schemes and their modal instances (i.e. W-instances).

2. WE is the axiom scheme $\mathrm{W} \varphi \rightarrow \neg \varphi$.

3. REW is the rule of extensionality: $\varphi \leftrightarrow \psi \vdash \mathrm{W} \varphi \leftrightarrow \mathrm{W} \psi$.

4. MP is the rule modus ponens: $\varphi, \varphi \rightarrow \psi \vdash \psi$.

\footnotetext{
${ }^{1}$ See https://arxiv.org/pdf/1707.03859.pdf
} 
The notion of syntactic consequence (i.e. $\vdash$ ) is rather standard: if $\Gamma$ is a set of $\mathbf{i E}^{\mathrm{W}}$-formulas, then $w \vdash \varphi$ iff $\varphi$ can be obtained from the finite subset of $\Gamma$ by using axioms of $\mathbf{i E}^{\mathrm{W}}$ and both inference rules. Clearly, if $\varphi \in \Gamma$, then $\Gamma \vdash \varphi$. The same concept of $\vdash$ will be accepted in the further systems.

The following theorem holds (and is simple to prove):

THEOREM 2.10. $\mathbf{i E}^{\mathrm{W}}$ is sound with respect to the class of all $\mathbf{i E}^{\mathrm{W}} \mathbf{p n}$ frames.

We can briefly prove completeness of our system with respect to the appropriate class of frames. For brevity, we assume that the reader is aware of the fact that each consistent $\mathbf{i E}^{\mathrm{W}}$-theory can be extended to the prime theory (this is just an intuitionistic version of the well-known Lindenbaum lemma). Hence, we may go directly to the canonical model.

For brevity, we start from the general pattern that will be used many times later.

Definition 2.11. iL $^{\mathrm{W}}$ can-pn-model is a triple $\langle W, \leq, \mathcal{N}, V\rangle$ where $\mathbf{L}$ may be any logic expressed in $\mathbf{i E}^{\mathrm{W}}$-language, and:

1. $W$ is the set of all prime theories of the logic $\mathbf{L}$.

2. For every $w, v \in W$ we say that $w \leq v$ iff $w \subseteq v$.

3. $\mathcal{N}$ is a function from $W$ into $P(P(W))$.

4. $V: P V \rightarrow P(W)$ is a function defined as it follows: $w \in V(q) \Leftrightarrow q \in$ $w$.

Later we shall use the following shortcut: $\widehat{\varphi}=\{z \in W ; \varphi \in z\}$. Now we may deal with the first particular case:

DeFinition 2.12. $\mathbf{i E}^{\mathrm{W}}$ can-pn-model is an $\mathbf{i L}^{\mathrm{W}}$ can-pn-model where $\mathbf{L}=$ $\mathbf{i E}^{\mathrm{W}}$ and for every $w \in W$ and for each formula $\varphi$ :

$\mathcal{N}_{w}=\{\widehat{\varphi} ; \mathrm{W} \varphi \in w\}$.

We need the following lemma:

LEMMA 2.13. $\mathbf{i E}^{\mathrm{W}}$ can-pn-model is indeed an $\mathbf{i} \mathbf{E}^{\mathrm{W}} \mathbf{p n}-$ model.

Proof: In fact, we must check that the monotonicity holds. Let us assume that $w \subseteq v$ and $v \notin X \in \mathcal{N}_{w}$. Now $X=\widehat{\varphi}$ for certain $\varphi$ such that $\mathrm{W} \varphi \in w$. However, $w$ is contained in $v$, hence $\mathbf{W} \varphi \in v$. Thus $\widehat{\varphi} \in \mathcal{N}_{v}$. 
Remark 2.14. Note that we did not use the fact that $v \notin X$. Actually, it was not important. For this reason, we may say that $\mathbf{i} \mathbf{E}^{\mathrm{W}}$ can-pn-model satisfies even stronger restriction, namely:

$$
\left[w \leq v, X \in \mathcal{N}_{w}\right] \Rightarrow X \in \mathcal{N}_{v} .
$$

Clearly, our completeness theorem will be true for this smaller class of frames (models). However, we may be interested not only in narrowing down these classes for which completeness result can be proved, but also in broadening those which are sufficient for the monotonicity of forcing.

Our neighborhood function is well-defined. First, if we assume that $W$ is a collection of all prime theories and $\widehat{\varphi}=\widehat{\psi}$, then we can easily prove that $\vdash \varphi \leftrightarrow \psi$ (using only non-modal tools). Second, assume that $\widehat{\varphi} \in \mathcal{N}_{w}$ and $\widehat{\varphi}=\widehat{\psi}$. If $\widehat{\varphi} \in \mathcal{N}_{w}$, then $\mathrm{W} \varphi \in w$. But $\varphi \leftrightarrow \psi \in \mathbf{i E}^{\mathrm{W}}$ (as we know from the first point of these considerations). Now, by means of REW, $\mathrm{W} \varphi \leftrightarrow \mathrm{W} \psi \in \mathbf{i E}^{\mathrm{W}} \subseteq w$. By MP, $\mathrm{W} \psi \in w$.

Our expected theorem about properties of the canonical model is below:

Theorem 2.15. Let $M=\langle W, \leq, \mathcal{N}, V\rangle$ be a $\mathbf{i E}^{\mathrm{W}}$ can-pn-model. Then for each $\gamma$ and for each $w \in W$ the following holds: $w \Vdash \gamma \Leftrightarrow \gamma \in w$.

Proof: Boolean cases are simple (of course we should remember that implication is intuitionistic). As for the modal case, let us assume that $\gamma=\mathrm{W} \varphi$.

$(\Rightarrow)$

Assume that $w \Vdash \gamma$. Hence, $w \Vdash \neg \varphi$ and $V(\varphi) \in \mathcal{N}_{w}$. By induction hypothesis $\widehat{\varphi} \in \mathcal{N}_{w}$. But then, by the very definition of canonical neighborhood, $\mathrm{W} \varphi \in w$.

Let $\mathrm{W} \varphi \in w$. By means of WE and MP we infer that $\neg \varphi \in w$. This is Boolean case: we are able to prove in a standard manner ${ }^{2}$ that $w \Vdash \neg \varphi$. From the definition of canonical neighborhood we have that $\widehat{\varphi} \in \mathcal{N}_{w}$. But $\widehat{\varphi}=\{z \in W ; \varphi \in z\}$. Now we use induction hypothesis (which is true in any world of our canonical model) to say that $\{z \in W ; \varphi \in z\}=\{z \in$ $W ; z \Vdash \varphi\}$. But the last set is precisely $V(\varphi)$. Hence $V(\varphi) \in \mathcal{N}_{w}$. We sum up our results to say that $w \Vdash \mathrm{W} \varphi$.

\footnotetext{
${ }^{2}$ We may use the fact that $\neg \varphi$ can be written as $\varphi \rightarrow \perp$.
} 
Now it is easy to formulate theorem about completeness:

THEOREM 2.16. $\mathbf{i E}^{\mathrm{W}}$ is strongly complete with respect to the class of all $\mathrm{iE}^{\mathrm{W}} \mathbf{p n}$-frames; and also with respect to those in which neighborhood function satisfies Cond. 2.2.

The proof of the theorem above is standard and based on the assumption that $w$ is a theory and $w \nvdash \varphi$. The idea is to show that there is a prime theory $v$ in a canonical model such that $w \subseteq v$ and $w \nVdash \varphi$.

One could say that our logic is not a proper system of "false belief" because it is (in its modal aspect) much weaker than some systems studied in [3], [2] and [1]. This will be discussed in the next subsection.

\subsection{Stronger systems of false belief}

It is not difficult to add one very natural axiom to our initial kit, namely WC : $(\mathrm{W} \varphi \wedge \mathrm{W} \psi) \rightarrow \mathrm{W}(\varphi \wedge \psi)$. Here are necessary definitions:

Definition 2.17. $\mathbf{i E C}^{\mathrm{W}}$ is defined as $\mathbf{i E}^{\mathrm{W}} \cup\{\mathrm{WC}\}$.

DeFinition 2.18. $\mathbf{i E C} \mathbf{C}^{\mathrm{W}} \mathbf{p n}$-model is defined as $\mathbf{i} \mathbf{E}^{\mathrm{W}} \mathbf{p n}$-model with one additional clause (the one of closure under binary intersections):

$$
\left[X, Y \in \mathcal{N}_{w}\right] \Rightarrow X \cap Y \in \mathcal{N}_{w} .
$$

Canonical model for $\mathbf{i E C} \mathbf{C}^{\mathrm{W}}$ (i.e. $\mathbf{i E C} \mathbf{C}^{\mathrm{W}}$ can-pn-model) is defined exactly in the same way as $\mathbf{i E}^{\mathrm{W}}$ can-pn-model (but its worlds are prime theories of $\mathbf{i E C}{ }^{\mathrm{W}}$ ). Thus, we should only prove the following lemma:

\section{LEMMA 2.19. $\mathbf{i E C}^{\mathrm{W}}$ can-pn-model is indeed an $\mathbf{i E C}^{\mathrm{W}} \mathbf{p n}-$ model.}

Proof: This is simple. Assume that $X, Y \in \mathcal{N}_{w}$. Hence, $X=\widehat{\varphi}$ and $Y=\widehat{\psi}$ (for certain $\varphi$ and $\psi$ such that $\mathrm{W} \varphi \in w$ and $\mathrm{W} \psi \in w$ ). Then $X \cap Y=\widehat{\varphi} \cap \widehat{\psi}=\widehat{\varphi \wedge \psi}$. At the same time, we use axiom WC to say that $\mathrm{W}(\varphi \wedge \psi) \in w$. Thus $X \cap Y \in \mathcal{N}_{w}$.

Now we can say that:

THEOREM 2.20. $\mathbf{i E C} \mathrm{W}^{\mathrm{W}}$ is strongly complete with respect to the class of all $\mathbf{i E C}^{\mathrm{W}} \mathbf{p n}$-frames (and those $\mathbf{i E C}{ }^{\mathrm{W}} \mathbf{p n}$-frames which satisfy Cond. 2.2). 
Let us introduce another system: it will be an intuitionistic version of $\mathbf{M}^{\mathrm{W}}$ studied in $[1] .^{3}$

DEFINITION 2.21. $\mathbf{i M}^{\mathrm{W}}$ is defined as $\mathbf{i E}^{\mathrm{W}} \cup\{\mathrm{RMW}\}$, where:

1. RMW is the rule $\varphi \rightarrow \psi \vdash(\mathrm{W} \varphi \wedge \neg \psi) \rightarrow \mathrm{W} \psi)$.

We introduce a new kind of models:

DEFinition 2.22. $\mathbf{i} \mathbf{M}^{\mathrm{W}} \mathbf{p n}$-model is an $\mathbf{i} \mathbf{E}^{\mathrm{W}} \mathbf{p n}$-model with one additional clause (the one of supplementation):

$$
\left[X \in \mathcal{N}_{w}, X \subseteq Y\right] \Rightarrow Y \in \mathcal{N}_{w} .
$$

LEMMA 2.23. $\mathbf{i} \mathbf{M}^{\mathrm{W}}$ is sound with respect to the class of all $\mathbf{i} \mathbf{M}^{\mathrm{W}} \mathbf{p n}$-models.

Proof: We shall check only RMW. Assume that $\varphi \rightarrow \psi$ is globally true. Suppose that there are $M=\langle W, \leq, \mathcal{N}, V\rangle$ and $w \in W$ such that $w \nVdash$ $(\mathrm{W} \varphi \wedge \neg \psi) \rightarrow \mathrm{W} \psi)$. Hence, there is $v \geq w$ such that $v \Vdash(\mathrm{W} \varphi \wedge \neg \psi)$ but $v \nVdash \mathrm{W} \psi$. This means that: i) $v \Vdash \neg \varphi, V(\varphi) \in \mathcal{N}_{v}, v \Vdash \neg \psi$; and ii) $v \nVdash \neg \psi$ or $V(\psi) \notin \mathcal{N}_{v}$. It is not possible that $v \nVdash \neg \psi$. On the other hand, if $\varphi \rightarrow \psi$ is globally true, then $V(\varphi) \subseteq V(\psi)$. Supplementation allows us to say that $V(\psi) \in \mathcal{N}_{v}$. This is contradiction.

Let us go to the canonical model.

DEFINITION 2.24. iM $^{\mathrm{W}}$ can-pn-model is an $\mathbf{i L}^{\mathrm{W}}$ can-pn-model where $\mathbf{L}=$ $\mathbf{i M}^{\mathrm{W}}$ and for every $w \in W$ and for each formula $\varphi$ :

$\mathcal{N}_{w}=\left\{X \subseteq W\right.$; there is $Y \in n_{w}$ such that $\left.Y \subseteq X\right\}$, where $n_{w}=$ $\{\widehat{\varphi} ; \mathrm{W} \varphi \in w\}$.

We must prove the following lemma:

LEMMA 2.25. $\mathbf{i} \mathbf{M}^{\mathrm{W}}$ can-pn-model is indeed an $\mathbf{i M}^{\mathrm{W}} \mathbf{p n}-$ model.

Proof: Let us think about monotonicity condition, namely Cond. 2.1. Assume that $w \subseteq v, v \notin X$ and $X \in \mathcal{N}_{w}$. Now there is $Y \in n_{w}$ such that $Y \subseteq X$. However, as we already know, function $n$ satisfies Cond. 2.2 which is even stronger than Cond. 2.1. Hence $Y \in n_{v}$ and thus $X \in \mathcal{N}_{v}$.

\footnotetext{
${ }^{3}$ Precisely speaking, Fan used axiom $\mathrm{W}(\varphi \wedge \psi) \wedge \neg \psi \rightarrow \mathrm{W} \psi$. The rule REW can be derived from this axiom and WE.
} 
As for the supplementation, it is obvious by the very definition of iM $^{\mathrm{W}}$ can-pn-model. Assume that $X \in \mathcal{N}_{w}$ and $X \subseteq Y$. Then there is $S \in n_{w}$ such that $S \subseteq X \subseteq Y$. We are ready.

Remark 2.26. Note that in fact $\mathbf{i M}^{\mathrm{W}}$ can-pn-model satisfies stronger monotonicity condition, i.e. Cond. 2.2. As in the case of $\mathbf{i E}^{\mathrm{W}}$ can-pn-model, we did not use the fact that $v \notin X$.

The next theorem is crucial for completeness:

TheOREM 2.27. Let $M=\langle W, \leq, \mathcal{N}, V\rangle$ be an $\mathbf{i M}^{\mathrm{W}}$ can-pn -model. Then for each $\gamma$ and for each $w \in W$ the following holds: $w \Vdash \gamma \Leftrightarrow \gamma \in w$.

Proof: We consider the modal case. Assume that $\gamma=\mathrm{W} \varphi$.

Let $w \Vdash \mathrm{W} \varphi$. Then $w \Vdash \neg \varphi$ and $V(\varphi) \in \mathcal{N}_{w}$. Hence $\neg \varphi \in w$ and $\widehat{\varphi} \in \mathcal{N}_{w}$. Also there is $\widehat{\psi} \in n_{w}$ such that $\widehat{\psi} \subseteq \widehat{\varphi}$ and $\mathrm{W} \psi \in w$. Now $\vdash \psi \rightarrow \varphi$, i.e. this formula is a theorem. Hence, (by means of RMW) $\vdash(\mathrm{W} \psi \wedge \neg \varphi) \rightarrow \mathrm{W} \varphi$. Assume now that $\mathrm{W} \varphi \notin w$. There are two possible reasons. First: $\boldsymbol{W} \psi \notin w$ (contradiction). Second: $\neg \varphi \notin w$. But $\neg \varphi \in w$, as we already know.

$(\Leftarrow)$

Assume that $\mathrm{W} \varphi \in w$. Now $\neg \varphi \in w$ and then $w \Vdash \neg \varphi$. Then $\widehat{\varphi} \in \mathcal{N}_{w}$. By induction hypothesis, $V(\varphi) \in \mathcal{N}_{w}$. Thus, $w \Vdash \mathrm{W} \varphi$.

THEOREM 2.28. $\mathbf{i M}^{\mathrm{W}}$ is strongly complete with respect to the class of all $\mathbf{i M}^{\mathrm{W}} \mathbf{p n}$-models (and those $\mathbf{i M}^{\mathrm{W}} \mathbf{p n}$-models which satisfy Cond. 2.2).

Let us sum up these results.

Definition 2.29. $\mathbf{i} \mathbf{K}^{\mathrm{W}}$ is defined as $\mathbf{i} \mathbf{M}^{\mathrm{W}} \cup\{\mathrm{WC}\}$.

DEFinition 2.30. $\mathbf{i} \mathbf{K}^{\mathrm{W}} \mathbf{p n}$-model is an $\mathbf{i} \mathbf{E}^{\mathrm{W}} \mathbf{p n}$-model satisfying both supplementation and closure under binary intersections.

Definition 2.31. $\mathbf{i K}^{\mathrm{W}}$ can-pn-model is defined just like $\mathbf{i M}^{\mathrm{W}} \mathbf{c a n}-\mathbf{p n}$-model (but $W$ consists of $\mathbf{i} \mathbf{K}^{\mathrm{W}}$ prime theories).

THEOREM 2.32. $\mathbf{i K}^{\mathrm{W}}$ is strongly complete with respect to the class of all supplemented $\mathbf{i} \mathbf{M}^{\mathrm{W}} \mathbf{p n}$-models closed under binary intersections (and those of them which satisfy Cond. 2.2). 
Proof: It is enough to check closure under intersections in iK ${ }^{\mathrm{W}}$ can-pn-model. Let $X, Y \in \mathcal{N}_{w}$. Hence, there are $\widehat{\varphi}, \widehat{\psi} \in n_{w}$ such that $\widehat{\varphi} \subseteq X$ and $\widehat{\psi} \subseteq Y$. Clearly, $\widehat{\varphi} \cap \widehat{\psi}=\widehat{\varphi \wedge \psi} \subseteq X \cap Y$. Also, by means of WC, $\mathbf{W}(\varphi \wedge \psi) \in w$ and thus $X \cap Y \in n_{w}$. Finally, $X \cap Y \in \mathcal{N}_{w}$.

There are some issues which should be discussed. This will be done in the next subsection.

\subsection{About canonical models and monotonicity}

In general, we borrowed some ideas from [1] and [2]. However, there are some subtle differences. Let us resume the line of thought presented in [1] with respect to the classical version of $\mathbf{i} \mathbf{M}^{\mathrm{W}}$, that is $\mathbf{M}^{\mathrm{W}}$.

i) Fan assumed that the canonical model for $\mathbf{M}^{\mathrm{W}}$ is any model based on maximal theories ${ }^{4}$ in which $\mathrm{W} \varphi \vee \varphi \in w \Leftrightarrow \widehat{\varphi} \in \mathcal{N}_{w}\left[{ }^{*}\right.$. Let us define also another condition for the further needs: $\mathrm{W} \varphi \in w \Leftrightarrow \widehat{\varphi} \in \mathcal{N}_{w}[* *]$.

Thus, he has defined the whole family of such models (from the minimal to the maximal one; the former contains precisely proof-sets ${ }^{5}$, the latter consists of proof-sets and all non-proof-sets).

Whereas we defined our $n_{w}$ (for any $w \in W$ ) precisely just like in the minimal model. We said that $n_{w}$ contains only those $\widehat{\varphi}$ for which $\mathrm{W} \varphi \in w$. Also, our line of reasoning was closer to [**] than to [*] but this is not crucial here.

ii) Then Fan introduced the notion of supplemented canonical model $M^{+}$(supplementation of canonical model $M$, in other words) in which $\mathcal{N}_{w}^{+}=\left\{X \subseteq W\right.$; there is $Y \in \mathcal{N}_{w}$ such that $\left.Y \subseteq X\right\}$. He showed that $M^{+}$is indeed canonical: that it satisfies [*]. Due to some reasons, it would be problematic for him to show that $M^{+}$satisfies [**]. It would require typical monotonicity rule $\varphi \rightarrow \psi \vdash \mathrm{W} \varphi \rightarrow \mathrm{W} \psi$ which is not sound.

Our way is different. We do not say that neighborhood function $\mathcal{N}$ in our $\mathbf{i M}^{\mathrm{W}}$ can-pn-model is "canonical" in the same sense as $n$. It would be irrelevant because the definition of $n_{w}$ leaves no place for any variants: as we said, these are precisely proof-sets satisfying certain property. However, maybe it would be sensible to follow Fan directly? Assume that $n_{w}$ is defined by means of, let us say, clause $\left[{ }^{* *}\right]$. It can be $[*]$ also, it does not

\footnotetext{
${ }^{4}$ With valuation defined as usual.

${ }^{5}$ Such proof-sets $\widehat{\varphi}$ that $\mathrm{W} \varphi \vee \varphi \in w$, of course.
} 
matter: the real problem lies in the persistence of truth. Both $[*]$ and $\left[{ }^{* *}\right]$ are too vague to force monotonicity (with respect to $n$ and in the sense of Cond. 2.1 or Cond. 2.2). Assume that $w \subseteq v$ and $X \in n_{w}$. We may also suppose that $v \notin X$. If $X=\widehat{\varphi}$ for certain $\varphi$, then we can repeat our actual reasoning. But if $X \neq \hat{\varphi}$ for any $\varphi$, then we cannot say anything special about this fact. Of course, if our model was maximal, then by the very definition $X$ would belong to $n_{v}$. But if not, then we would be in a quandary.

It seems that a similar solution to a similar dilemma has been obtained in [4]. Recall that these authors prepared bi-neighborhood semantics for weak intuitionistic modal logics and they also used minimal canonical models. In case of richer logics they used canonical models "equipped with" supplementation (just as our $\mathbf{i} \mathbf{M}^{\mathrm{W}}$ can-pn-model), not the supplementation of previously defined model.

Gilbert and Venturi found different solution than Fan did. They assumed that neighborhoods in canonical model (for the classical version of $\mathbf{i K}^{\mathrm{W}}$ ) are defined by means of [**]. Then they used negative supplementation. This is the following condition:

$$
Y \in \mathcal{N}_{w}, \quad Y \subseteq X, \quad w \notin X \Rightarrow X \in \mathcal{N}_{w}
$$

In the negative supplementation of canonical model, for any $w \in W$ and for each $\varphi$ we have:

$$
\mathcal{N}_{w}^{+}=\left\{X \subseteq W \text {; there is } Y \in \mathcal{N}_{w} \text { such that } Y \subseteq X \text { and } w \notin X\right\} .
$$

Again, this "feature of negativity" (that is, the assumption that $w \notin X$ ) is helpful in proving that negative supplementation is indeed canonical. From our point of view, one thing is interesting. Let us reproduce the definition of $\mathbf{i K}^{\mathrm{W}}$ can-pn-model but with the following definition of neighborhoods:

$$
\mathcal{N}_{w}=\left\{X \subseteq W ; \text { there is } Y \in n_{w} \text { such that } Y \subseteq X \text { and } w \notin X\right\},
$$

where $n_{w}=\{\widehat{\varphi} ; \mathrm{W} \varphi \in w\}$.

This is in accordance with our previous considerations. The whole proof of completeness is almost identical. However, there is one noteworthy moment. Let us prove that Cond. 2.1 of monotonicity is satisfied. Let $w \subseteq v, X \in \mathcal{N}_{w}$ and $v \notin X$. There is $Y \in n_{w}$ such that $Y \subseteq X$. However, 
$n$ satisfies Cond. 2.2 (Lem. 2.13 and Rem. 2.14), so $Y \in n_{v}$. Thus $X \in \mathcal{N}_{v}$. Note that in this case we prove only that $\mathcal{N}$ satisfies Cond. 2.1 and not necessarily Cond. 2.2. Clearly, we used the assumption that $v \notin X$.

\section{Conclusion and future work}

In this paper we have discussed several false belief systems based on the intuitionistic core. In general, these results are rather natural. However, it does not mean that all of them are straightforward. We have pointed out some nuances and compared our results with those of other authors.

Logics of false belief are often investigated together with the logics of unknown truths. It seems that this line of research is more complicated. Classical versions of these systems were introduced by Steinsvold in [3]. Neighborhood semantics for them has been presented by Gilbert and Venturi in [2]. Some new results on this matter have been later obtained by Fan in [1].

The very idea of uknown truth is that a formula is true but not known. This is expressed by the following interpretation of the modal operator

$$
w \Vdash \bullet \varphi \Leftrightarrow w \Vdash \varphi \text { and } V(\varphi) \notin \mathcal{N}_{w} .
$$

We may also say that $\varphi$ is accepted (by our agent) but it is not suggested by his "advisory board".

As for the operator $\bullet$, it can be used interchangeably with $\circ$ which is defined as below:

$$
w \Vdash \circ \varphi \Leftrightarrow w \nVdash \varphi \text { or } V(\varphi) \in \mathcal{N}_{w} \Leftrightarrow \text { if } w \Vdash \varphi \text { then } V(\varphi) \in \mathcal{N}_{w} .
$$

In a classical setting we may identify $\circ$ with $\neg \bullet \varphi$ and $\bullet$ with $\neg \circ \varphi$. The authors mentioned above have already proved completeness of several systems based on $\circ$ (or, equivalently, $\bullet$ ).

Things become more complex when our logic is intuitionistic. There are at least three problems: monotonicity of $\circ^{6}$; interchangeability of

\footnotetext{
${ }^{6}$ Assume that $w \Vdash \circ \varphi$. It can mean that $w \nVdash \varphi$. Just as in the case of our earlier operator $\mathrm{W}$, it is not reasonable to expect that $\varphi$ will be rejected in each $v \geq w$. It would be difficult (if at all possible) to impose an appropriate condition on frames. One possible solution is to replace rejection of $\varphi$ with an acceptance of $\neg \varphi$. However, this does not give us mutual duality of $\circ$ and $\bullet$, at least not the same as in the classical system.
} 
and $\circ^{7}$; soundness and completeness. We shall not discuss these issues here: they deserve more detailed studies which we consider as our future work.

Finally, we think that it would be natural to connect (both classical and intuitionistic) logics of false belief (or / and unknown truths) with some paraconsistent tools. Actually, we think about operators of indeterminacy $(N)$ and ambiguity $(M)$, invented and investigated by Żabski in [5]. Basically, Żabski assumed that valuation $V$ connects each formula $\varphi$ with 0 or 1 . Now $V(N \varphi)=1 \Leftrightarrow V(\varphi)=0$ and $V(\neg \varphi)=0$, while $V(M \varphi)=1$ $\Leftrightarrow V(\varphi)=0$ and $V(\neg \varphi)=0$. Of course negation is not classical or intuitionistic here. Rather, it is paraconsistent. Note that it makes sense, from the philosophical point of view, to model the following situation: $\varphi$ is undetermined or ambiguous (e.g. in a given world $w$ ), yet it is believed (or suggested by our advisors). We have already made some (unpublished) attempts in this direction, also in the quasi-intuitionistic setting (namely, in paraconsistent models with persistence of truth).

Acknowledgements. We are grateful to our anonymous reviewers for their comments which helped us to reformulate the structure of our paper (and some particular statements or definitions).

\section{References}

[1] J. Fan, Notes on neighborhood semantics for logics of unknown truths and false beliefs (2020), arXiv:2002.09622, math.LO.

[2] D. Gilbert, G. Venturi, Neighborhood Semantics for Logics of Unknown Truths and False Beliefs, The Australasian Journal of Logic, vol. 14(1) (2017), pp. 246-267, DOI: https://doi.org/10.26686/ajl.v14i1.4033.

[3] C. Steinsvold, Being Wrong: Logics for False Belief, Notre Dame Journal of Formal Logic, vol. 52(3) (2011), pp. 245-253, DOI: https://doi.org/10. 1215/00294527-1435438.

${ }^{7}$ One could say that partial or total lack of duality would not be bad in this case. Note that mutual independence of $\square$ and $\diamond$ is a rather desired property in intuitionistic modal logic. This approach can be transferred to the logics of unknown truths. Moreover, we may always limit our attention to only one of these operators. 
[4] N. O. T. Dalmonte, Ch. Grellois, Intuitionistic non-normal modal logics: A general framework, Journal of Philosophical Logic, vol. 49 (2020), pp. 833-882, DOI: https://doi.org/10.1007/s10992-019-09539-3.

[5] E. Żabski, Logiki nihilistyczne, czyli teorie prawd "powierzchownych" i "głebokich", Oficyna Wydawnicza Politechniki Wrocławskiej, Wrocław (2001).

\section{Tomasz Witczak}

University of Silesia

Faculty of Science and Technology

Institute of Mathematics

Bankowa 14, 40-007 Katowice, Poland

e-mail: tm.witczak@gmail.com 